\title{
Immunohistochemistry as alternative to DNA sequencing in detection of BRAF V600E mutation in papillary thyroid carcinomas
}

Paja $\mathrm{M}^{1}$, del Cura $\mathrm{JL}^{2}$, Pérez de Ciriza $\mathrm{M}^{1}$, Oleaga $\mathrm{A}^{1}$, Ugalde $\mathrm{A}^{3}$.

Endocrinology ${ }^{1}$, Interventional Radiology ${ }^{2}$ and Pathology Department ${ }^{3}$. Basurto University Hospital. Bilbao. Spain.

\section{Introduction:}

BRAF V600E mutation is the most common genetic alteration in papillary thyroid carcinomas (PTC) and is associated with worse prognosis. Recently, new ATA guidelines have pointed out its relevance at risk stratification in patients with PTC. Direct sequencing is usually performed, but less expensive immunohistochemistry can be used instead. We compare the results of both techniques in a series of patients with CPT.

\section{Methods/Design:}

Study was performed in 54 consecutive PTCs during 2014-2015. DNA was extracted from formalin-fixed paraffin-embedded tissues by manual-microdissection and BRAF mutation was detected by allele specific PCR with Cobas ${ }^{\circledR} 4800$ BRAF V600 mutation test (Roche). IHC was performed over tissue fixed for 24-h with $10 \%$ neutral formalin using the anti-BRAF V600E (VE-1) mouse monoclonal primary antibody. IHC was scored as positive or negative at pathologist's criteria.

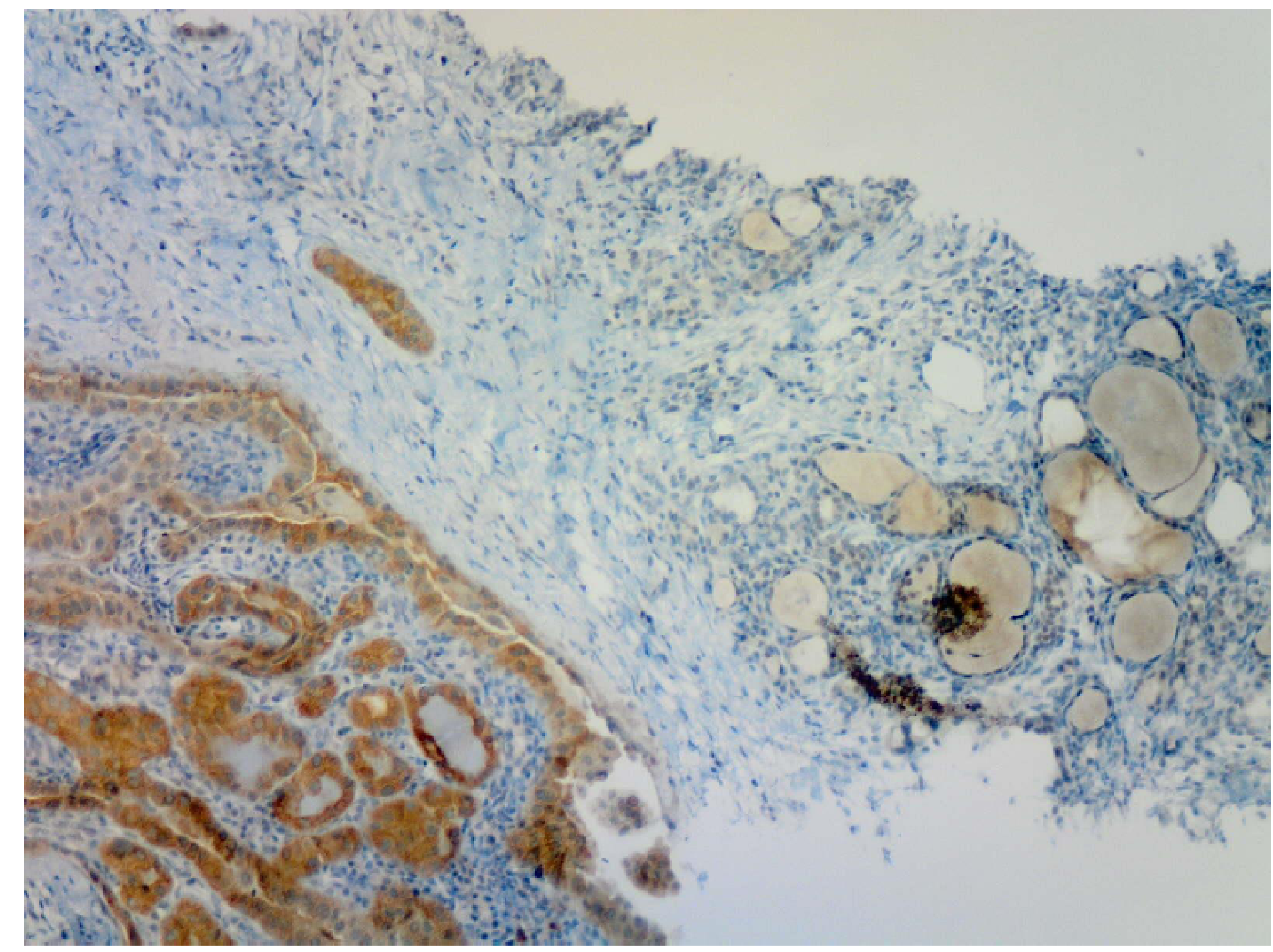

IHC BRAF positive CPT next to normal IHC negative thyroid tissue

IHC BRAF negative CPT

\section{Results:}

IHC BRAF V600E was positive in 32 PTC and negative in 22. Mutated PTC appeared in older patients (mean: 53.2 vs 50.8 yo). There were 18 positive tumors by IHC with multifocality $(56.2 \%)$ whereas only 7 of $22(31.8 \%)$ with negative $\mathrm{IHC}$ had two or more foci. Negative IHC PTCs were bigger than positive ones (17.3 vs $12.8 \mathrm{~mm}$ ). Half of positive IHC cases were classic variants of PTC, but only one in negative IHC neoplasms. Of the 32 cases that were IHC positive for BRAF V600E, 31 were confirmed by sequencing $(96.9 \%)$ and one case was discordant. Discordance appeared in a 38 year-old woman with an incidental PTC follicular variant of $5 \mathrm{~mm}$, near to a follicular adenoma with suspicious FNA. Pathologist confirmed that tissue had been taken from the tumor.
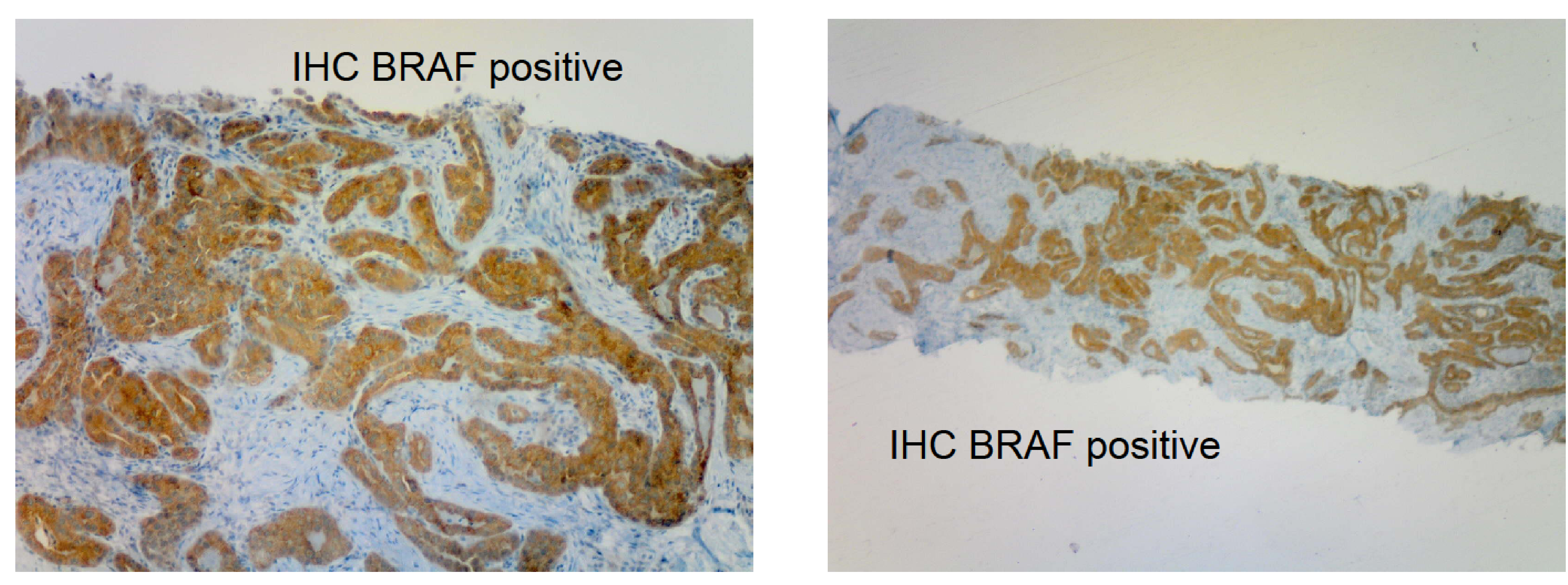

IHC BRAF positive

\section{Conclusion}

IHC has an accuracy of $96.9 \%$ for BRAF V600E detection and can be used as costeffective DNA sequencing in daily practice. 